Supplement of Atmos. Chem. Phys., 14, 4909-4934, 2014

http://www.atmos-chem-phys.net/acp-14-4909-2014/

doi:10.5194/acp-14-4909-2014-supplement

(c) Author(s) 2014. CC Attribution 3.0 License.

(c) (1)

Supplement of

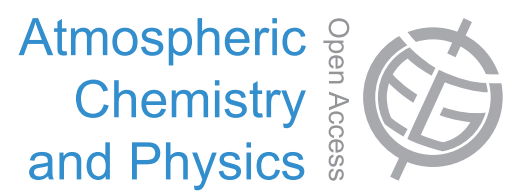

\title{
Strong wintertime ozone events in the Upper Green River basin, Wyoming
}

B. Rappenglück et al.

Correspondence to: B. Rappenglück (brappenglueck@uh.edu) 
1 S1: Overview of air quality instrumentation used in this study (1-min data available, unless noted otherwise). At all sites standard meteorological 2 systems.

\begin{tabular}{|c|c|c|c|c|c|}
\hline $\begin{array}{l}\text { Boulder site } \\
42.7186^{\circ} \mathrm{N},-109.7531^{\circ} \mathrm{W} ; \\
2,160 \mathrm{~m} \text { asl }\end{array}$ & Method & Model & Accuracy & Precision & $\begin{array}{l}\text { Detection } \\
\text { Limit }\end{array}$ \\
\hline $\mathrm{O}_{3}$ & UV Photometric & T-API 400E & & $0.5 \%$ & $0.6 \mathrm{ppbv}$ \\
\hline $\mathrm{NO}^{*++)}$ & Chemiluminescence & Air Quality Design, Inc 2-channel NO detector & $\pm 5.4 \%+3 \mathrm{pptv}$ & & $3 p p t v$ \\
\hline $\mathrm{NO}_{2}^{*,+)}$ & Chemiluminescence & $\begin{array}{l}\text { Air Quality Design, Inc 2-channel NO detector } \\
\text { with photolytic converter (BLC) }\end{array}$ & $\pm 13.6 \%+6 \mathrm{pptv}$ & & $6 \mathrm{pptv}$ \\
\hline $\mathrm{NO}_{\mathrm{y}}^{*,++}$ & Chemiluminescence & $\begin{array}{l}\text { Air Quality Design, Inc 2-channel NO detector } \\
\text { with molybdenum catalytic converter }\end{array}$ & $\pm 14.8 \%+4 \mathrm{pptv}$ & & 4pptv \\
\hline $\mathrm{HNO}_{3}{ }^{\#}$ & $\begin{array}{l}\text { Redox Denuder Difference, } \\
\text { chemiluminescence detector }\end{array}$ & $\begin{array}{l}\text { Custom-built instrument by Air Quality design. } \\
\text { Includes use of a T-API model 200EU } \\
\text { chemiluminescence NO detector }\end{array}$ & $\pm 26.9 \%+50 \mathrm{pptv}$ & & $0.1 \mathrm{ppbv}$ \\
\hline HONO $\left.^{* *}\right)$ & Long Path Absorption Photometry & QUMA-LOPAP & $\pm 10 \%$ & $5 \%$ & $1-2 \mathrm{pptv}$ \\
\hline $\mathrm{HCHO}^{* * *)}$ & Fluorometric Hantzsch Reaction & AL 4021 & $\pm 2 \%$ & $10 \%$ & $60 \mathrm{pptv}$ \\
\hline $\mathrm{NMHC} / \mathrm{CH}_{4}{ }^{1)}$ & Flame Ionization Detection & Baseline-Mocon Series $9000 \mathrm{NMHC} / \mathrm{CH}_{4}$ analyzer & $\pm 5 \%$ & $\pm 5 \%$ & $0.1 \mathrm{ppmv}$ \\
\hline Speciated particulates ${ }^{2)}$ & $\begin{array}{l}\text { IMPROVE_A/TORTOT } \\
\text { Elements: X-ray Fluorescence } \\
\text { Major ions: Ion Chromatography }\end{array}$ & $\begin{array}{l}\text { URG-3000N Carbon Sampler } \\
\text { Met One SASS Speciation Air Sampler System }\end{array}$ & $\begin{array}{l}\text { Total OC: } 2-6 \% \\
\text { Total EC: } 2-6 \% \\
<10 \%\end{array}$ & $\begin{array}{l}<10 \% \\
<10 \%\end{array}$ & $\begin{array}{l}0.45 \mu \mathrm{g} / \mathrm{cm}^{2} \\
0.06 \mu \mathrm{g} / \mathrm{cm}^{2}\end{array}$ \\
\hline Mixing Layer height ${ }^{3)}$ & Doppler Sodar & ASC Model 4000 miniSodar & & & $15 \mathrm{~m}$ \\
\hline Radiosondes $^{3)}$ & $\begin{array}{l}\text { GPS-based Upper Air Sounding } \\
\text { System }\end{array}$ & InterMet iMet-3050 403 MHz GPS & $\begin{array}{l} \pm 0.5 \mathrm{~m} / \mathrm{s} \text { (horiz.) } \\
\pm 5^{\circ} \text { (horiz.) } \\
\pm 0.2^{\circ} \mathrm{C} \\
\pm 2 \%\end{array}$ & & \\
\hline Ozone sondes ${ }^{3)}$ & Titration of ozone in KI & EN-SCI Corp. KZ-ECC $\mathrm{O}_{3}$ sondes & $\pm 10 \%$ & & $2-3$ ppbv \\
\hline \multicolumn{6}{|l|}{$\begin{array}{l}\text { Boulder South Road } \\
42.6840^{\circ} \mathrm{N},-109.7083^{\circ} \mathrm{W} ; \\
2139 \mathrm{~m} \text { asl }\end{array}$} \\
\hline $\mathrm{NO}_{\mathrm{x}}{ }^{1)}$ & UV Photometric & Thermo Scientific 42i & $< \pm 5 \%$ & $<5 \%$ & $0.4 \mathrm{ppbv}$ \\
\hline $\mathrm{CO}^{1)}$ & NDIR & Thermo Scientific 48i TLE & $< \pm 5 \%$ & $<10 \%$ & $0.04 \mathrm{ppmv}$ \\
\hline Speciated NMHCs $^{1)}$ & GC/FID & Perkin Elmer Ozone Precursor Analyzer & $< \pm 3 \%$ & $<5 \%$ & $0.01 \mathrm{ppbv}$ \\
\hline
\end{tabular}




\begin{tabular}{|c|c|c|c|c|c|}
\hline $\begin{array}{l}\text { Tethered Balloo } \\
42.6822^{\circ} \mathrm{N},-109.8089^{\circ} \\
2,143 \mathrm{~m} \text { asl }\end{array}$ & & & & & \\
\hline Temperature $^{3)}$ & & HOBO U23 Pro V.2 & $\begin{array}{l} \pm 0.21^{\circ} \mathrm{C}\left(0^{\circ} \text { to } 50^{\circ} \mathrm{C}\right) \\
\pm 0.28^{\circ} \mathrm{C}\left(\text { at }-20^{\circ} \mathrm{C}\right) \\
\pm 0.5^{\circ} \mathrm{C}\left(\text { at }-30^{\circ} \mathrm{C}\right)\end{array}$ & & \\
\hline Relative Humidity ${ }^{3)}$ & & HOBO U23 Pro V.2 & $\begin{array}{c} \pm 2.5 \%(10 \%-90 \% \mathrm{RH}) \\
\pm 4.5 \% \text { (at } 100 \% \mathrm{RH})\end{array}$ & & \\
\hline $\mathrm{O}_{3}^{3,4)}$ & UV Photometric & T-API 400E & & $0.5 \%$ & $0.6 \mathrm{ppbv}$ \\
\hline $\mathrm{NO} / \mathrm{NO}_{2} / \mathrm{NO}_{\mathrm{x}}^{3,4)}$ & Chemiluminescence & T-API 200E & & $\begin{array}{l}0.5 \%>50 \\
\mathrm{ppb}\end{array}$ & $0.4 \mathrm{ppbv}$ \\
\hline $\mathrm{NMHC} / \mathrm{CH}_{4}^{3,4)}$ & Flame Ionization Detection & Baseline-Mocon Series $9000 \mathrm{NMHC} / \mathrm{CH}_{4}$ analyzer & $\pm 5 \%$ & $\pm 5 \%$ & $0.1 \mathrm{ppmv}$ \\
\hline
\end{tabular}

1) hourly data, 2) available as integrated $24 \mathrm{~h}$ measurements, 3) available on IOP days

4) surface-based instrumentation collecting data through a system of solenoid valves in the balloon inlet package which allowed the measurements to cycle between tethersonde height levels $(4 \mathrm{~m}, 33 \mathrm{~m}, 67 \mathrm{~m}$, and $100 \mathrm{~m})$ and provided a measurement at each level every 12 minutes.

*) Ridley and Grahek (1990); Reidmiller et al. (2010), **) Heland et al., 2001; Kleffmann et al., 2002, Ródenas et al., 2011, ***) Rappenglück et al., 2010

+ The Air Quality Design, Inc chemiluminescence instrument is a two-channel, high performance NO detector that uses a remote-mounted inlet system which houses the catalytic and photolytic converters. The instrument was operated with one channel time-sharing between the $\mathrm{NO}$ and $\mathrm{NO}_{\mathrm{x}}\left(\mathrm{NO}_{+} \mathrm{NO}_{2}\right)$ measurements, and one channel continuously measuring the $\mathrm{NO}_{\mathrm{y}}$ signal. The instrument was calibrated using a custom-built gas-phase titration calibration system that provided sensitivity measurements for both $\mathrm{NO}$ and $\mathrm{NO}_{2}$ channels. In addition, the $\mathrm{NO}_{y}$ channel was priodically challenged with a permeation-tube based $\mathrm{HNO}_{3}$ calibration source that was in place for calibration of the $\mathrm{HNO}_{3}$ instrument to ensure that the $\mathrm{NO}_{\mathrm{y}}$ converter was providing quantitative conversion of $\mathrm{HNO}_{3}$. Standard addition calibration was used in all cases to ensure that the measured sensitivities were representative of the ambient sample matrix. The $\mathrm{NO}_{\mathrm{xy}}$ remote inlet was mounted on the roof of the instrument enclosure at a height of about $3 \mathrm{~m}$ from the surface.

\# The $\mathrm{HNO}_{3}$ measurement system was comprised of a commercial chemiluminescence NO detector with a remote mounted inlet system. The inlet system included a twochannel molybdenum catalytic $\mathrm{NO}_{\mathrm{y}}$ converter, one of which also included a calcium carbonate coated denuder. The difference in signal between the two-channels represented the ambient $\left[\mathrm{HNO}_{3}\right]$. The instrument was calibrated using the same $\mathrm{NO}, \mathrm{NO}_{2}, \mathrm{HNO}_{3}$ calibration source utilized for the $\mathrm{NO}_{\mathrm{xy}}$ instrument. The $\mathrm{HNO}$ instrument inlet was colocated with the $\mathrm{NO}_{\mathrm{xy}}$ inlet at $3 \mathrm{~m}$ above the surface. 
S2. Results for selected time periods for the Boulder site. Data in [ppbv], except for $\mathrm{CH}_{4}$ and NMHC [ppmv]. Hourly data presented.

\section{IOP days}

26

\begin{tabular}{|c|c|c|c|c|c|c|c|c|c|c|c|c|}
\hline & \multicolumn{4}{|c|}{ 0500-0900 MST } & \multicolumn{4}{|c|}{ 1100-1700 MST } & \multicolumn{4}{|c|}{ 2100-0500 MST } \\
\hline & $\mathbf{Q}_{1}{ }^{*}$ & $\mathbf{Q}_{2}^{\#}$ & $\mathbf{Q}_{3}^{+}$ & Max. & $\mathbf{Q}_{1}{ }^{*}$ & $\mathbf{Q}_{2}^{\#}$ & $\mathbf{Q}_{3}^{+}$ & Max. & $\mathbf{Q}_{1}{ }^{*}$ & $\mathbf{Q}_{2}^{\#}$ & $\mathbf{Q}_{3}^{+}$ & Max. \\
\hline $\mathbf{O}_{3}$ & 42.7 & 46.6 & 48.7 & 50.9 & 63.2 & 73.0 & 119.6 & 165.8 & 46.3 & 51.0 & 58.7 & 87.7 \\
\hline NO & 0.019 & 0.310 & 1.408 & 23.535 & 0.315 & 0.575 & 2.093 & 18.681 & $\mathrm{BDL}$ & 0.002 & 0.012 & 0.550 \\
\hline $\mathrm{NO}_{2}$ & 1.416 & 5.362 & 10.862 & 25.815 & 0.791 & 1.863 & 3.229 & 29.012 & 1.899 & 3.353 & 9.748 & 29.648 \\
\hline $\mathbf{N O}_{\mathbf{x}}$ & 1.523 & 7.107 & 13.457 & 49.350 & 1.213 & 2.710 & 5.757 & 42.367 & 1.904 & 3.355 & 9.757 & 29.666 \\
\hline $\mathrm{NO}_{\mathbf{y}}$ & 3.045 & 9.587 & 15.977 & 55.398 & 5.902 & 11.287 & 19.498 & 66.443 & 4.914 & 7.848 & 13.170 & 30.493 \\
\hline $\mathrm{HNO}_{3}$ & 0.283 & 0.893 & 2.549 & 10.906 & 1.225 & 2.484 & 5.951 & 15.795 & 0.481 & 1.295 & 2.477 & 6.525 \\
\hline HONO & 0.130 & 0.317 & 0.442 & 1.360 & 0.201 & 0.510 & 0.972 & 1.397 & 0.093 & 0.164 & 0.363 & 0.908 \\
\hline НСНО & 0.100 & 0.401 & 0.603 & 2.180 & 0.295 & 0.855 & 1.252 & 1.995 & 0.137 & 0.313 & 0.577 & 1.182 \\
\hline $\mathrm{CH}_{4}$ & 2.00 & 2.65 & 3.40 & 9.30 & 2.00 & 2.80 & 3.90 & 6.1. & 2.00 & 2.30 & 2.80 & 6.10 \\
\hline NMHC & 0.10 & 0.45 & 0.73 & 1.20 & 0.20 & 0.60 & 1.20 & 2.20 & 0.20 & 0.30 & 0.50 & 2.00 \\
\hline
\end{tabular}

$\left.27 \quad{ }^{*}\right) \mathrm{Q}_{1}$ : first quartile $\left(25^{\text {th }}\right.$ percentile)

$29 \quad{ }^{\#)} \mathrm{Q}_{2}$ : second quartile (median)

$30 \quad{ }^{+)} \mathrm{Q}_{3}$ : third quartile $\left(75^{\text {th }}\right.$ percentile)

31 BDL: below detection limit 
S2. continued.

non-IOP days

\begin{tabular}{|c|c|c|c|c|c|c|c|c|c|c|c|c|}
\hline & \multicolumn{4}{|c|}{ 0500-0900 MST } & \multicolumn{4}{|c|}{$1100-1700$ MST } & \multicolumn{4}{|c|}{ 2100-0500 MST } \\
\hline & $\mathbf{Q}_{1}{ }^{*}$ & $\mathbf{Q}_{2}^{\#}$ & $\mathbf{Q}_{3}^{+}$ & Max. & $\mathbf{Q}_{1}^{*}$ & $\mathbf{Q}_{2}^{\#}$ & $\mathbf{Q}_{3}^{+}$ & Max. & $\mathbf{Q}_{1}{ }^{*}$ & $\mathbf{Q}_{2}^{\#}$ & $\mathbf{Q}_{3}^{+}$ & Max. \\
\hline $\mathbf{O}_{3}$ & 40.1 & 45.4 & 49.1 & 59.5 & 53.3 & 65.0 & 81.9 & 116.4 & 47.6 & 50.0 & 53.6 & 77.4 \\
\hline NO & 0.038 & 0.399 & 1.778 & 6.591 & 0.181 & 0.672 & 1.376 & 20.737 & BDL & 0.002 & 0.007 & 0.053 \\
\hline $\mathrm{NO}_{2}$ & 2.199 & 3.399 & 5.804 & 22.054 & 0.573 & 0.970 & 2.366 & 17.994 & 0.953 & 1.516 & 2.420 & 9.674 \\
\hline $\mathrm{NO}_{\mathrm{x}}$ & 2.255 & 4.455 & 6.243 & 25.725 & 0.817 & 1.617 & 3.685 & 38.730 & 0.962 & 1.516 & 2.446 & 9.677 \\
\hline $\mathrm{NO}_{\mathrm{y}}$ & 4.300 & 7.961 & 10.569 & 26.068 & 4.460 & 7.226 & 13.191 & 43.660 & 3.401 & 4.381 & 6.621 & 12.383 \\
\hline $\mathrm{HNO}_{3}$ & 0.509 & 1.044 & 1.300 & 4.383 & 0.682 & 1.295 & 3.050 & 8.556 & 0.180 & 0.544 & 0.869 & 2.398 \\
\hline HONO & 0.062 & 0.150 & 0.255 & 0.590 & 0.065 & 0.135 & 0.250 & 1.030 & 0.045 & 0.083 & 0.128 & 0.628 \\
\hline HCHO & 0.126 & 0.209 & 0.360 & 0.926 & 0.219 & 0.316 & 0.593 & 1.346 & 0.110 & 0.191 & 0.286 & 1.105 \\
\hline $\mathbf{C H}_{4}$ & 2.00 & 2.25 & 2.65 & 4.30 & 2.00 & 2.25 & 2.80 & 6.10 & 2.00 & 2.10 & 2.30 & 4.30 \\
\hline NMHC & 0.10 & 0.20 & 5.25 & 1.40 & 0.10 & 0.25 & 0.60 & 1.60 & 0.10 & 0.20 & 0.30 & 1.00 \\
\hline $\begin{array}{l}\left.{ }^{*}\right) \mathrm{Q}_{1} \text { : first } \\
{ }^{\#)} \mathrm{Q}_{2} \text { : seco } \\
{ }^{+)} \mathrm{Q}_{3} \text { : thirc } \\
\text { BDL: bel }\end{array}$ & $\begin{array}{l}\text { th }^{\text {th }} \text { perc } \\
\text { (media } \\
5^{\text {th }} \text { per } \\
\text { ion lin }\end{array}$ & & & & & & & & & & & \\
\hline
\end{tabular}



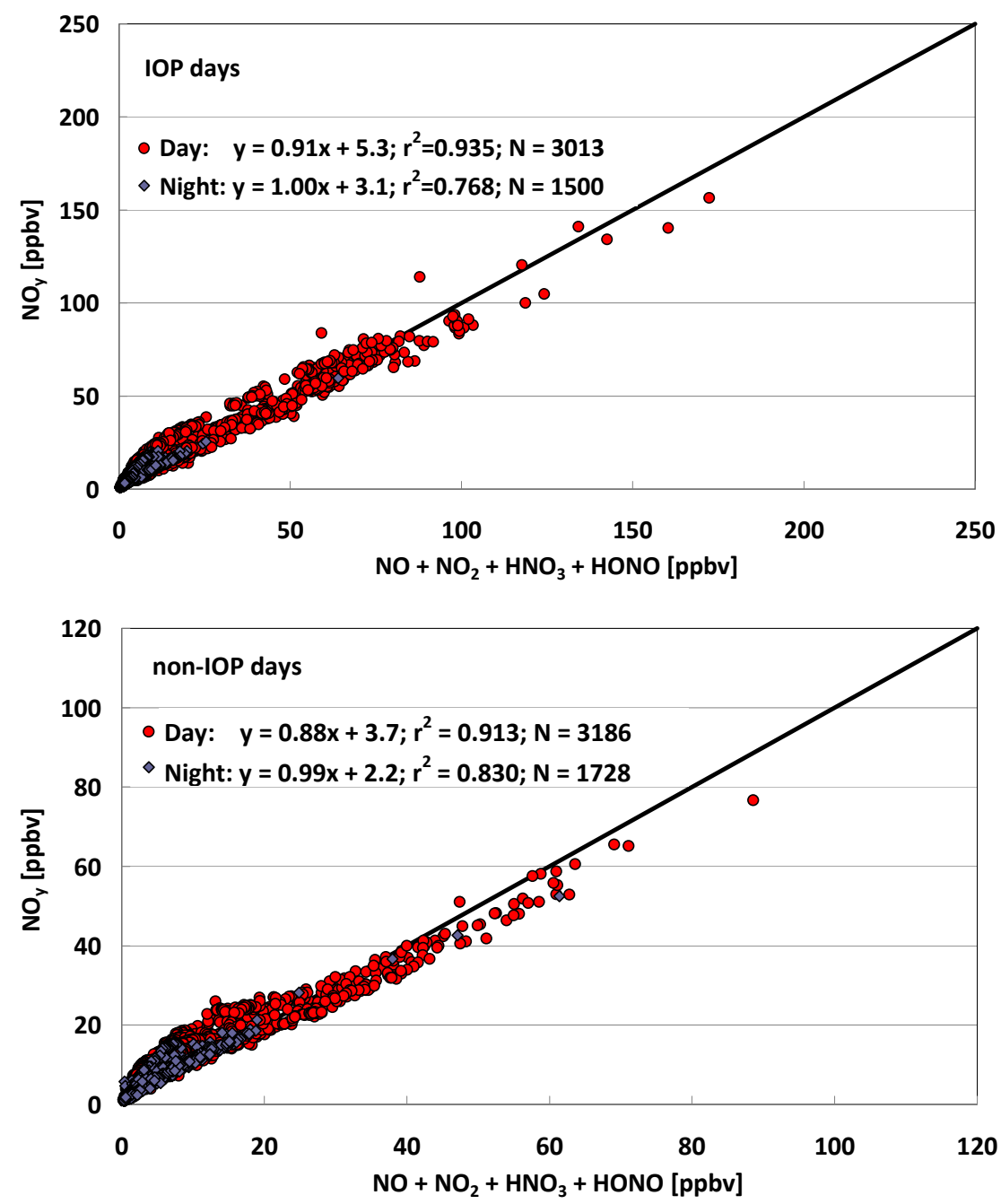

46

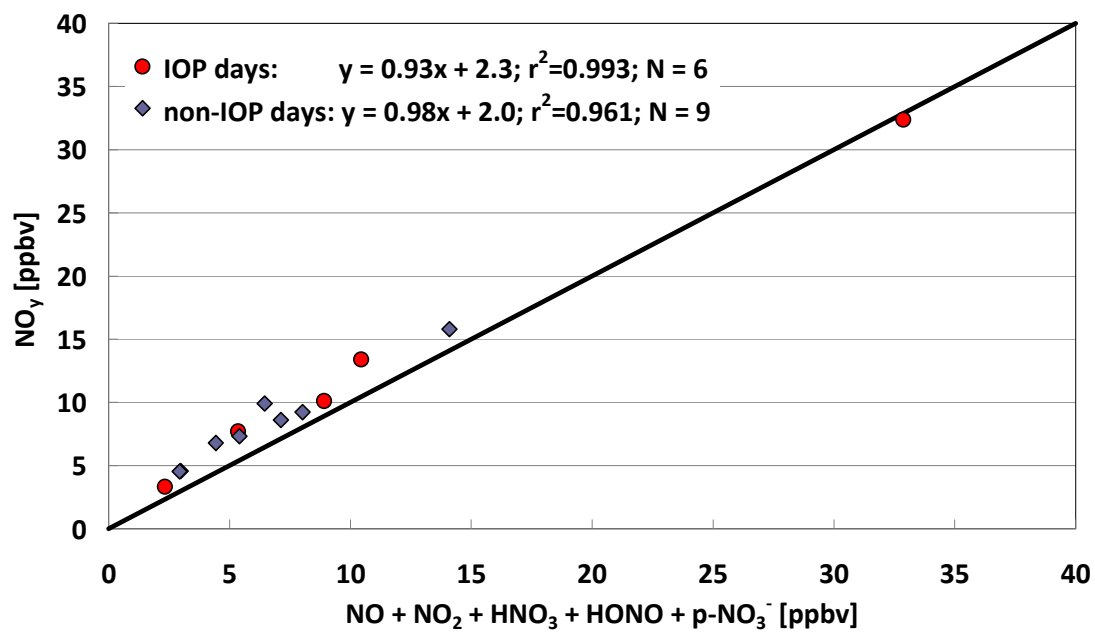

47

48 S3. $\mathrm{NO}_{\mathrm{y}}$ mixing ratios versus sum of mixing ratios of individual $\mathrm{NO}_{\mathrm{y}}$ compounds $\mathrm{NO}, \mathrm{NO}_{2}$, $\mathrm{HNO}_{3}, \mathrm{HONO}$, and particulate $\mathrm{NO}_{3}$. $\mathrm{N}$ denotes number of data points. 
50
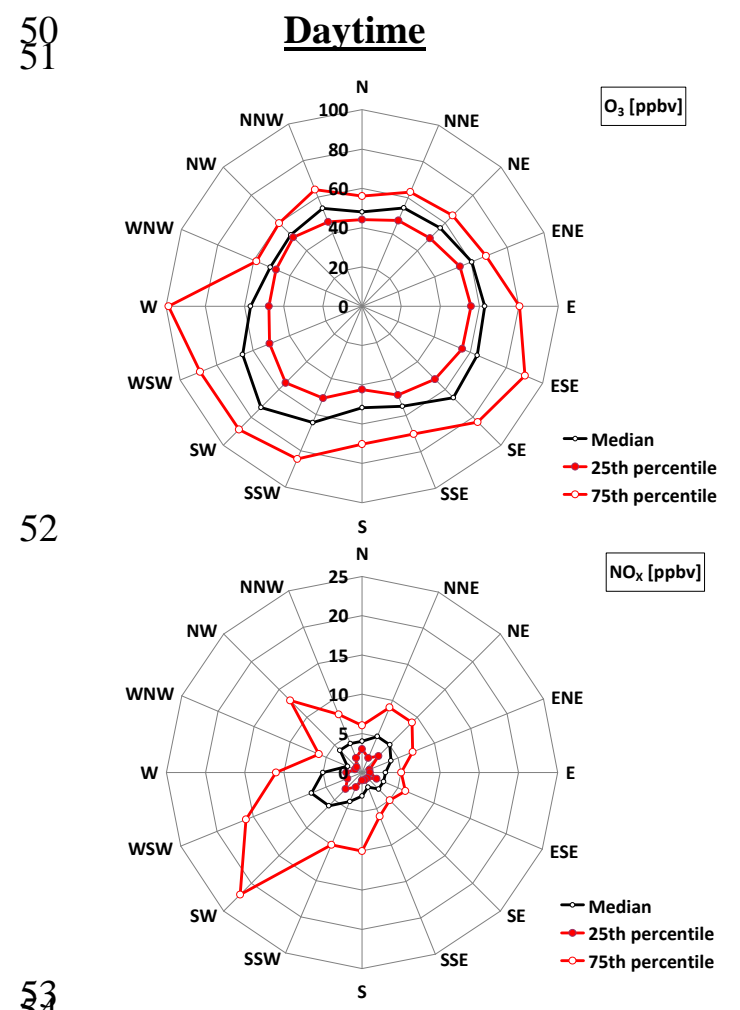

53

55
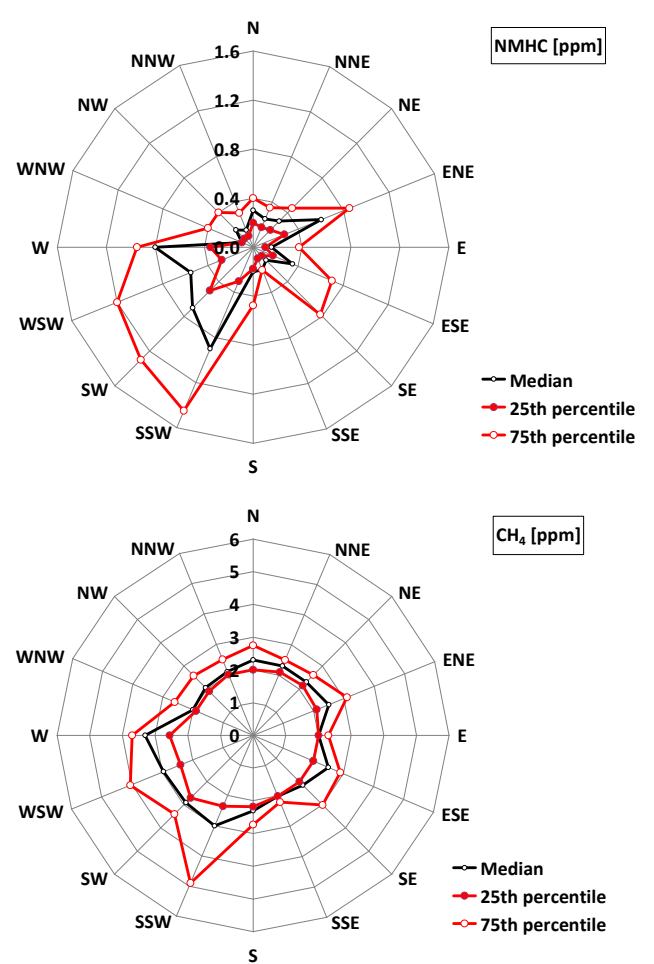

58

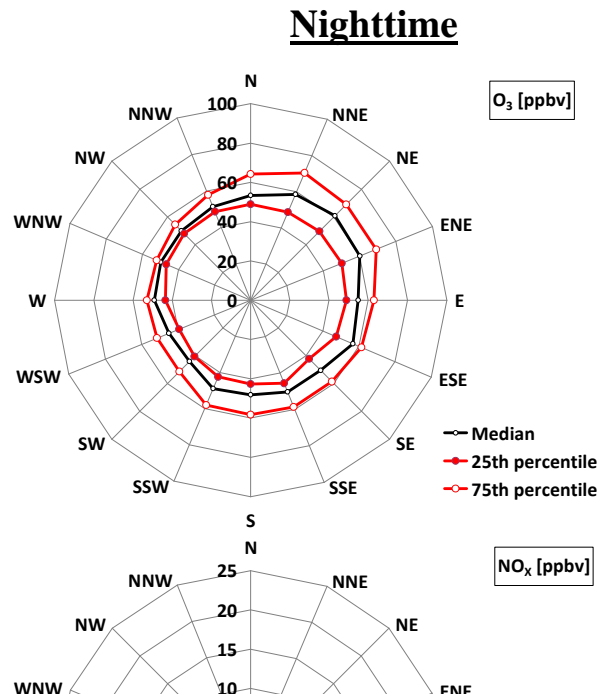

61
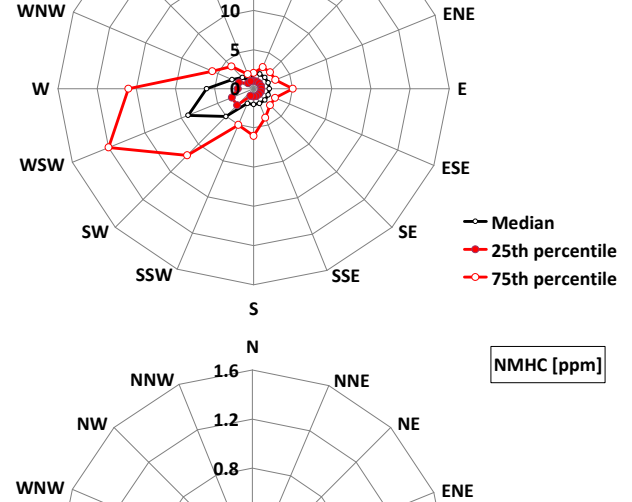

62
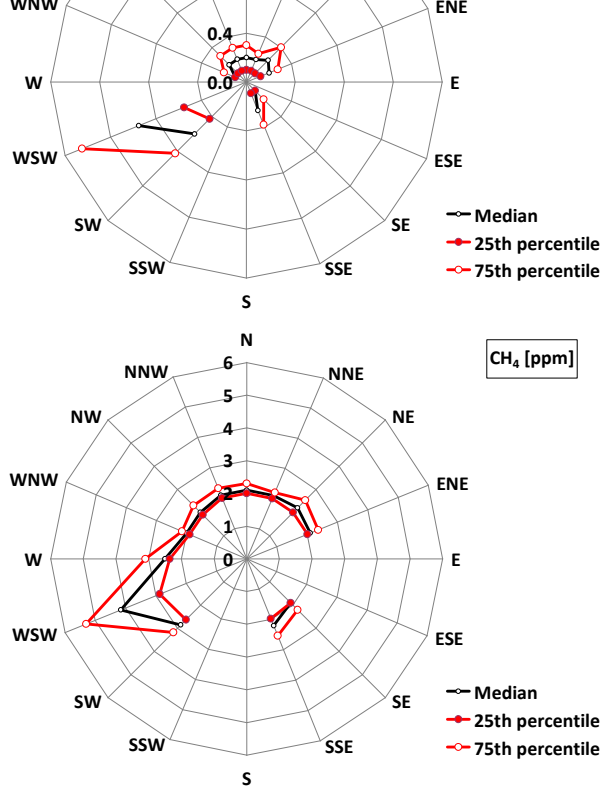

64 S4a. Wind directional dependence of selected trace gases for day- and night-time conditions

65 (night-time defined as time periods with solar radiation less than $1 \mathrm{~W} / \mathrm{m}^{2}$ ). Units shown in 66 brackets refer to the radial direction of the corresponding trace gas plot. 
68

69

70

71

72
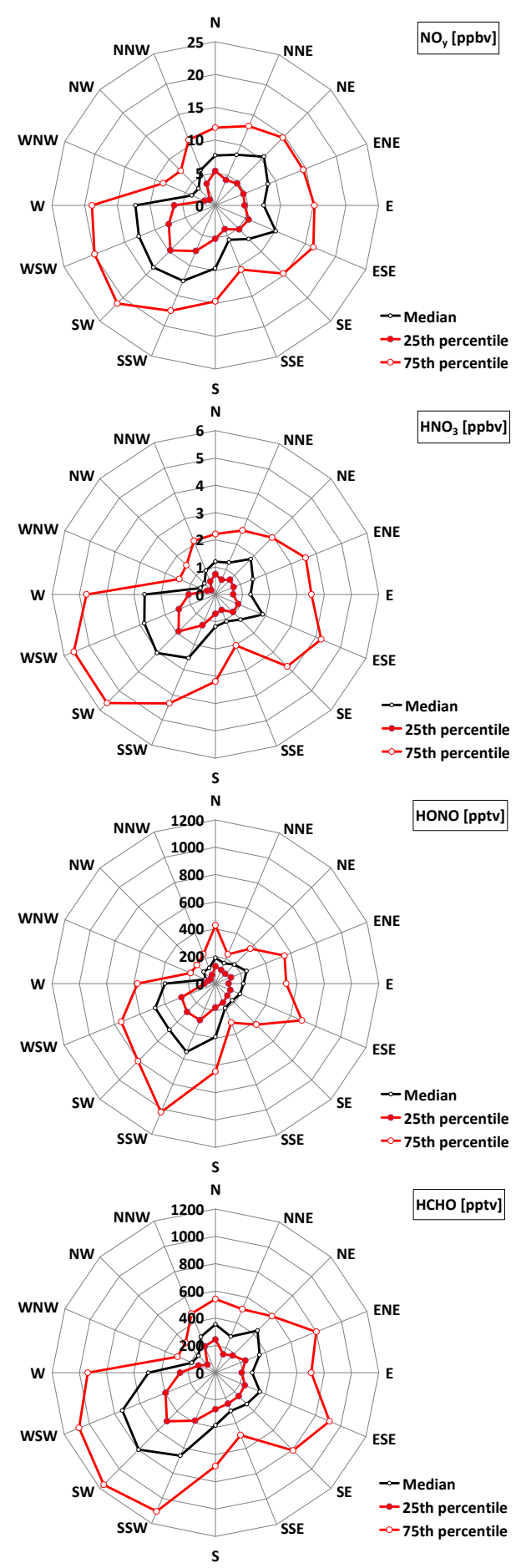

75

76

73
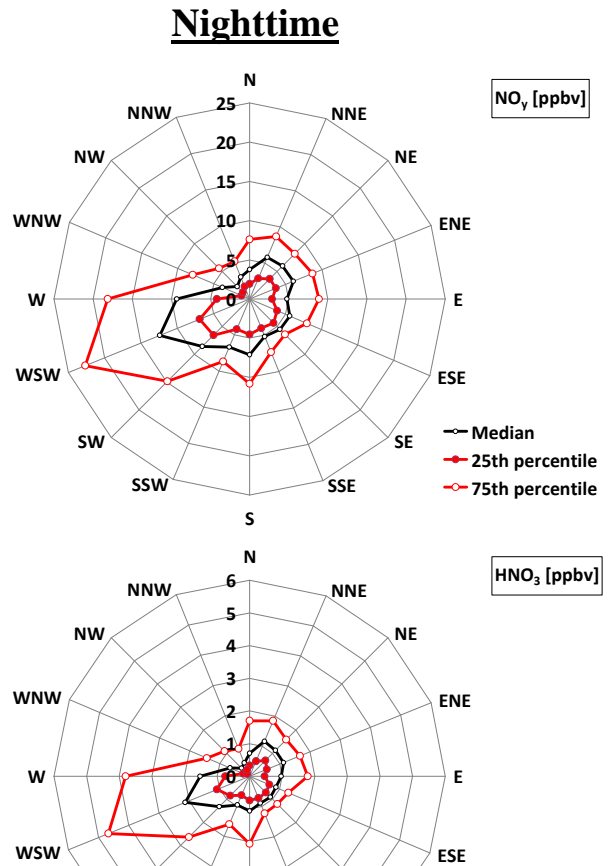

WSW

sw
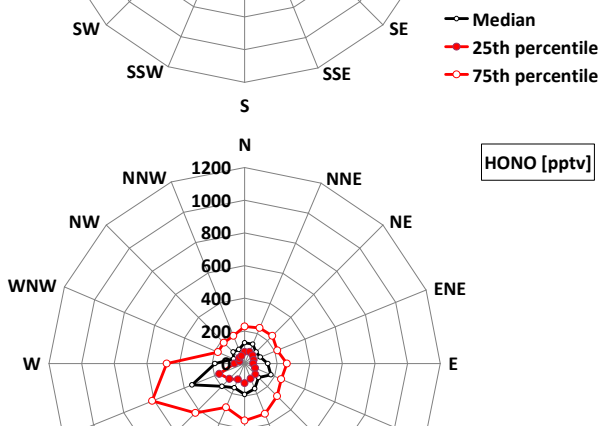

wsw

ESE

77

78

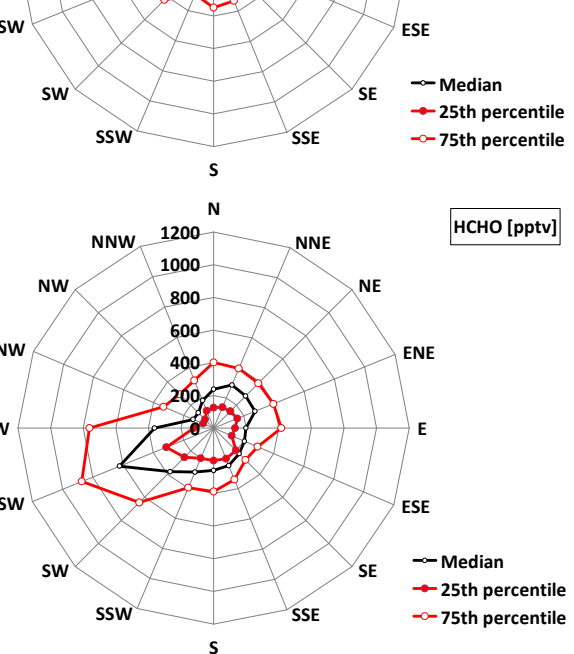

79 S4b. Wind directional dependence of selected trace gases for day- and night-time conditions

80 (night-time defined as time periods with solar radiation less than $1 \mathrm{~W} / \mathrm{m}^{2}$ ). Units shown in

81 brackets refer to the radial direction of the corresponding trace gas plot. 
83

84

85

86

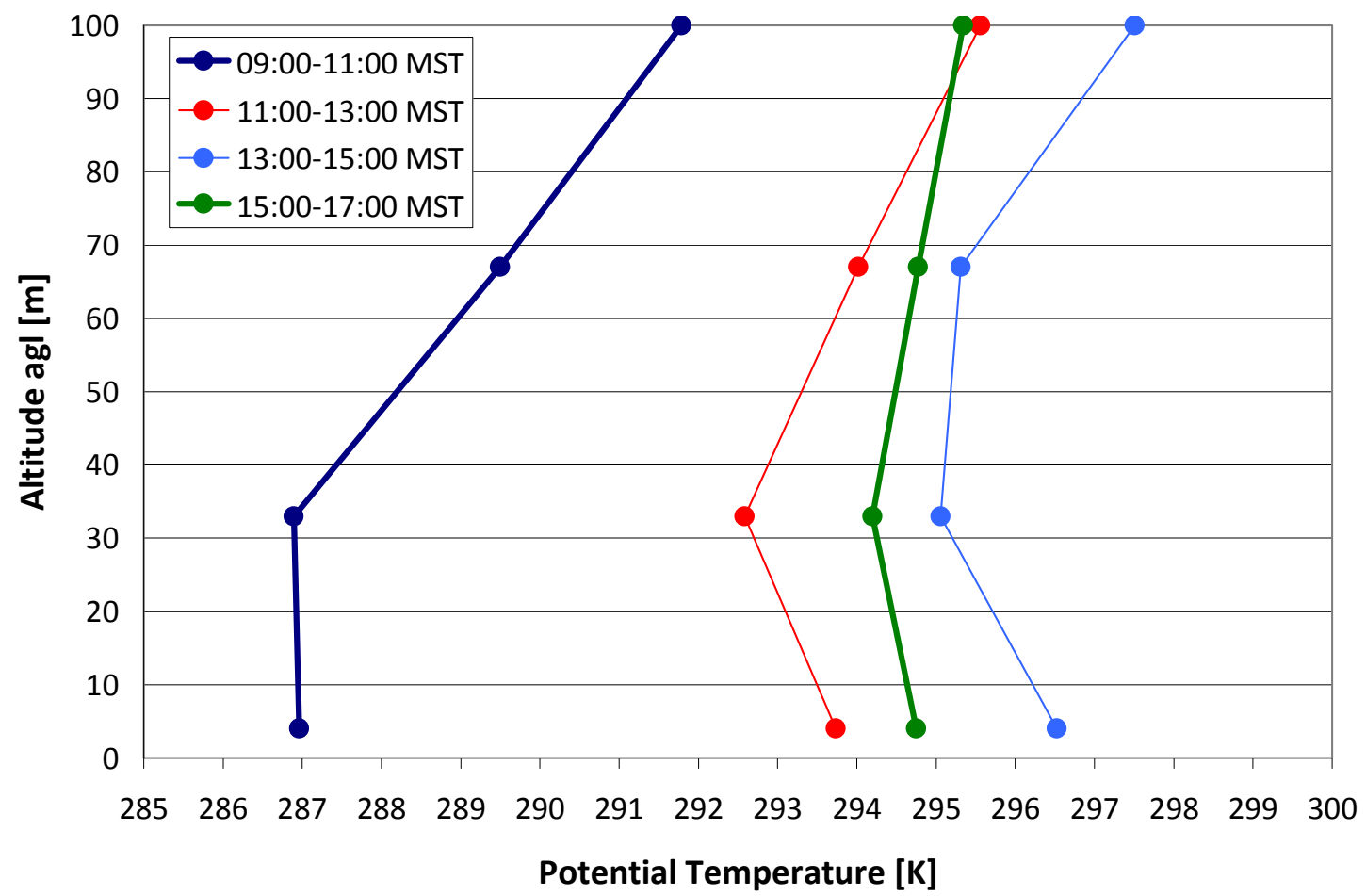

87

88

89 S5. Potential temperature profiles on IOP days based on tethersonde measurements segregated 90 into selected time frames.

91

92 


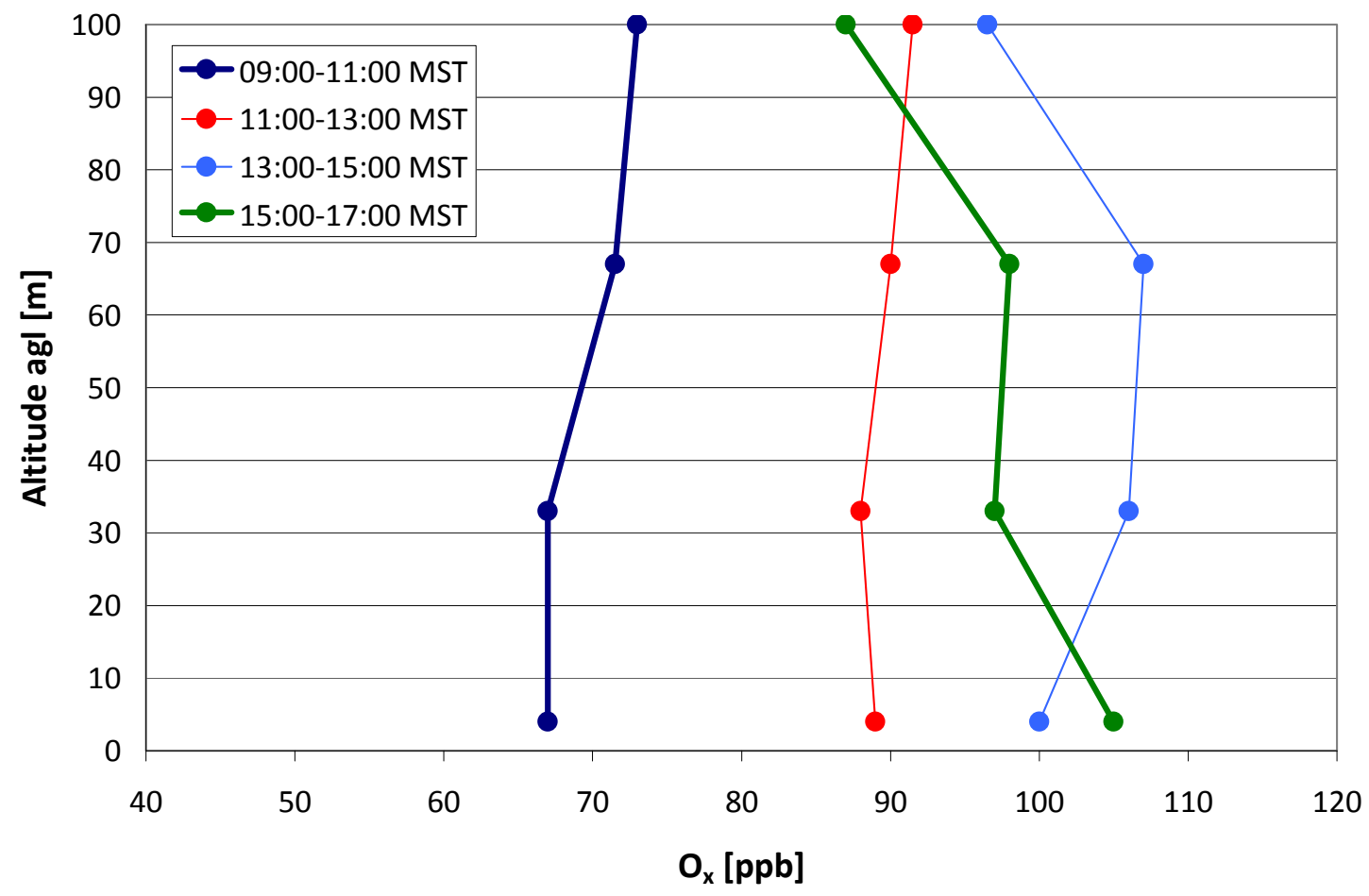

99 S6. Profiles of potential ozone $\mathrm{O}_{\mathrm{x}}\left(\mathrm{O}_{\mathrm{x}}=\mathrm{O}_{3}+\mathrm{NO}_{2}\right)$ on IOP days based on tethersonde 100 measurements segregated into selected time frames.

101

102

103 
104

105

Daytime

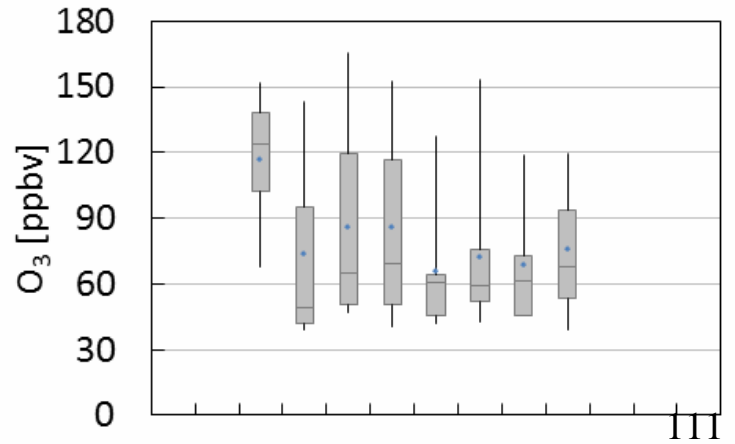

106

107

108

109
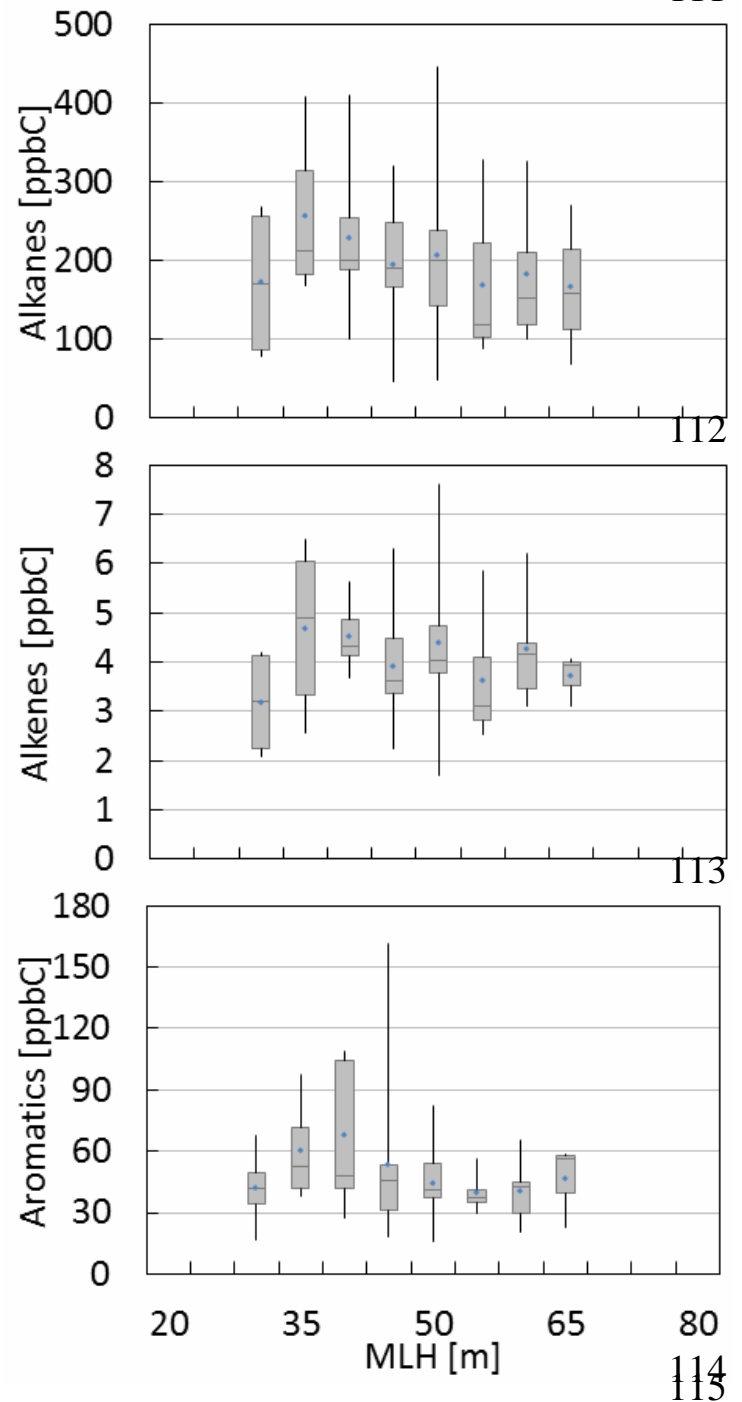

110
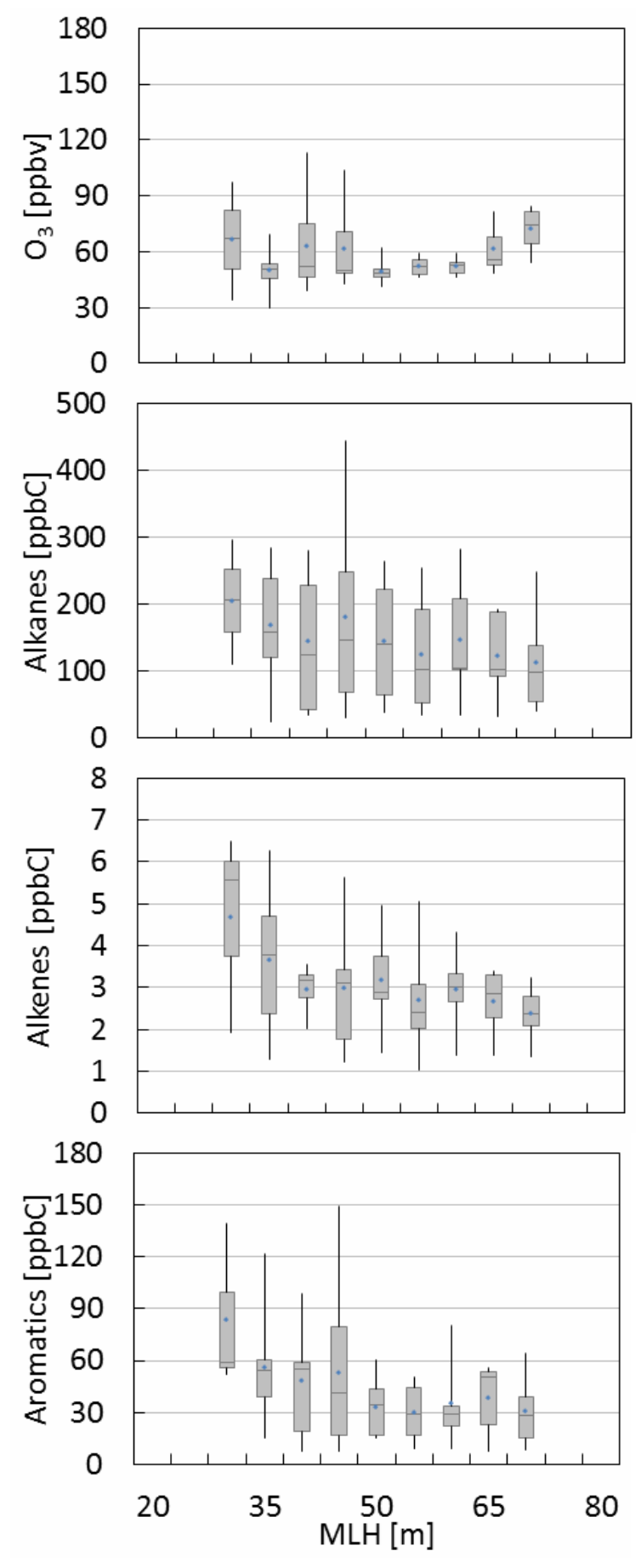

116 S7. Selected trace gas mixing ratios versus mixing layer heights (MLH) for day- and night-

117 time conditions on IOP days (night-time defined as time periods with solar radiation less than

$1181 \mathrm{~W} / \mathrm{m}^{2}$ ). Speciated NMHC, $\mathrm{CO}$, and $\mathrm{NO}_{\mathrm{x}}$ data from the Boulder South Road site. 
120

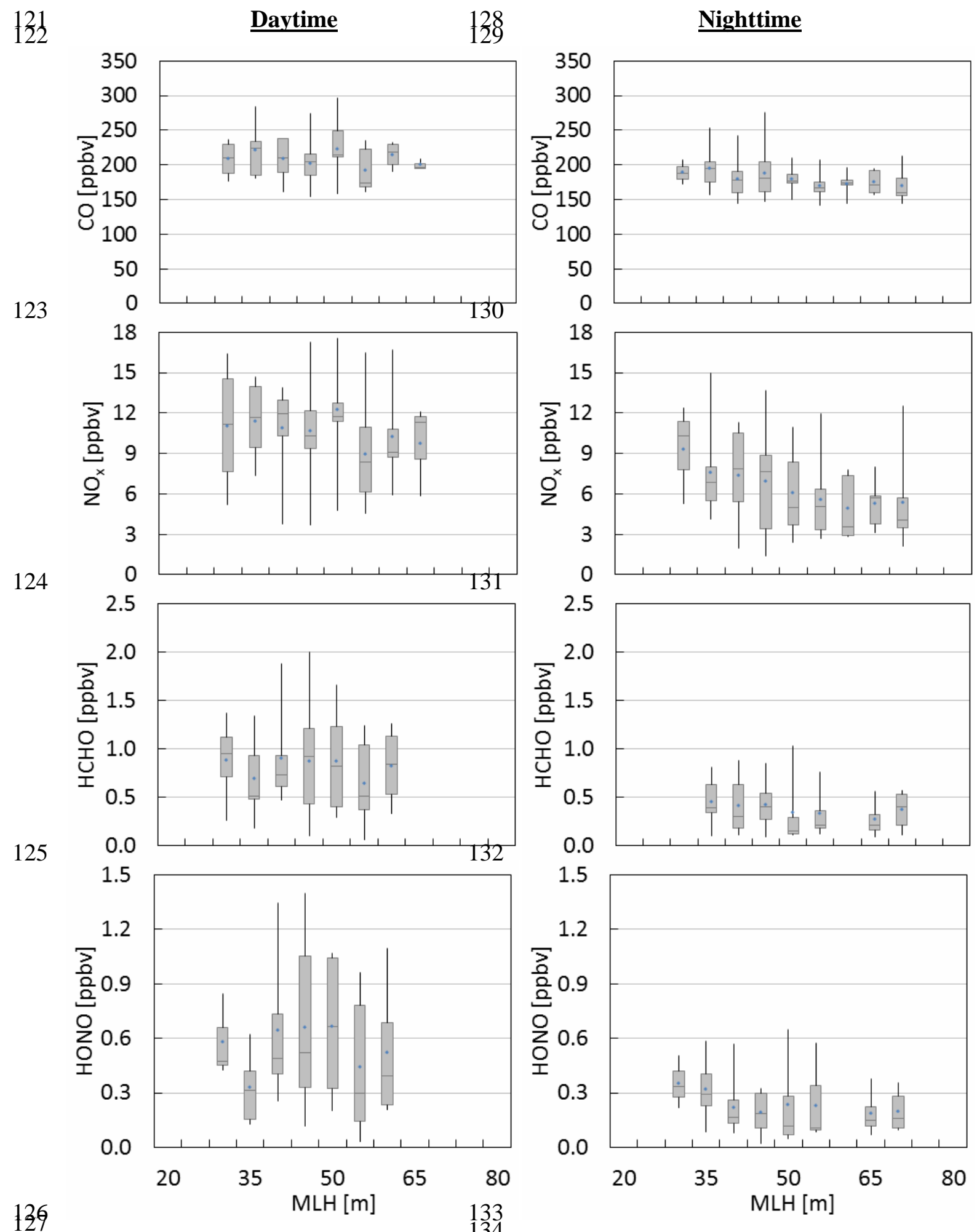

135 S7. continued. 
137 S8. Results of correlation analysis of HCHO and HONO versus selected other trace gases at 138 the Boulder site for nighttime conditions and wind direction $180^{\circ}-270^{\circ}$. All data $10-\mathrm{min}$ 139 values, apart from correlations which include $\mathrm{NMHC}$ or $\mathrm{CH}_{4}$, which are hourly values.

140

141

142

143

144

145

146

147

\section{5} 46

\begin{tabular}{|c|c|c|c|}
\hline HCHO vs CH & $\left.\mathrm{a}^{*}\right)$ & $\left.\mathrm{b}^{* *}\right)$ & $\mathrm{r}^{2}$ \\
\hline HCHO vs NMHC & $\begin{array}{c}0.223 \\
( \pm 0.040) \\
( \pm 0.098)\end{array}$ & $\begin{array}{c}-148.4 \\
( \pm 158.7)\end{array}$ & 0.71 \\
\hline HCHO vs $\mathrm{NO}_{\mathrm{x}}$ & $\begin{array}{c}15.44 \\
( \pm 1.45)\end{array}$ & $\begin{array}{c}324.7 \\
( \pm 31.2)\end{array}$ & 0.66 \\
\hline HONO vs $\mathrm{CH}_{4}$ & $\begin{array}{c}0.188 \\
( \pm 0.036)\end{array}$ & $\begin{array}{c}-225.9 \\
( \pm 138.5\end{array}$ & 0.70 \\
\hline HONO vs $\mathrm{NMHC}$ & $\begin{array}{c}0.441 \\
( \pm 0.070)\end{array}$ & $\begin{array}{c}91.2 \\
( \pm 69.2)\end{array}$ & 0.77 \\
\hline HONO vs $\mathrm{NO}_{2}$ & $\begin{array}{c}16.82 \\
( \pm 0.95)\end{array}$ & $\begin{array}{c}148.5 \\
( \pm 19.1)\end{array}$ & 0.80 \\
\hline HONO vs $\mathrm{NO}_{\mathrm{x}}$ & $\begin{array}{c}15.20 \\
( \pm 0.88)\end{array}$ & $\begin{array}{c}161.5 \\
( \pm 19.1)\end{array}$ & 0.79 \\
\hline $\begin{array}{c}100.92 \\
( \pm 5.95)\end{array}$ & $\begin{array}{c}88.1 \\
( \pm 22.6)\end{array}$ & 0.80 \\
\hline
\end{tabular}

*) a: slope in [pptv/ppbv]

$\left.{ }^{* *}\right)$ b: intercept in [pptv] 
149 S9. Values of indicator ratios for $\mathrm{NO}_{\mathrm{x}}$-sensitive, transitional, and VOC-sensitive conditions according to Sillman (2002) and Sillman and He (2002). Photochemical indicators for the moderatley polluted case (80 ppbv $<\mathrm{O}_{3}<200 \mathrm{ppbv}$ ), unless otherwise stated.

152

153

\begin{tabular}{|c|c|c|c|c|}
\hline Indicator & $\begin{array}{c}\text { Median VOC } \\
\text { sensitive }\end{array}$ & $\begin{array}{c}\text { Transition } \\
\left(\mathbf{O}_{3}<\mathbf{8 0} \mathbf{p p b v}\right)\end{array}$ & Transition & $\begin{array}{c}\text { Median } \mathbf{N O}_{\mathbf{x}} \\
\text { sensitive }\end{array}$ \\
\hline $\mathrm{O}_{3} / \mathrm{NO}_{\mathrm{y}}$ & 5 & $11-15$ & $6-8$ & 11 \\
\hline $\mathrm{O}_{3} / \mathrm{NO}_{\mathrm{z}}$ & 6 & $15-20$ & $8-10$ & 14 \\
\hline $\mathrm{O}_{3} / \mathrm{HNO}_{3}$ & 9 & n.a. $^{* *}$ & $12-15$ & 20 \\
\hline $\left.\mathrm{EOR}^{*}\right)$ & $\mathrm{EOR}<0.6$ & n.a. $^{* *}$ & $0.6<\mathrm{EOR}<0.9$ & $\mathrm{EOR}>0.9$ \\
\hline
\end{tabular}

154

*) Extent of Reaction

156

${ }^{* *}$ no information given by Sillman and He (2002), but presumably higher than for the moderately polluted case.

S10. Average minimum values of photochemical indicators at the Boulder site and time

163

164

\begin{tabular}{|c|c|c|}
\hline Indicator & $\begin{array}{c}\text { Average minimum median } \\
\text { values and time of occurrence }\end{array}$ & $\begin{array}{c}\text { Average minimum median values } \\
\text { and wind direction of occurrence }\end{array}$ \\
\hline $\mathrm{O}_{3} / \mathrm{NO}_{\mathrm{y}}$ & $3.1(09: 00 \mathrm{MST})$ & $3.2(\mathrm{SSW})$ \\
\hline $\mathrm{O}_{3} / \mathrm{NO}_{\mathrm{z}}$ & $10.1(14: 00 \mathrm{MST})$ & $9.2(\mathrm{SSW})$ \\
\hline $\mathrm{O}_{3} / \mathrm{HNO}_{3}$ & $14.1(09: 00 \mathrm{MST})$ & $11.0(\mathrm{~W})$ \\
\hline $\left.\mathrm{EOR}^{*}\right)$ & $0.53(08: 00 \mathrm{MST})$ & $0.55(\mathrm{WSW})$ \\
\hline
\end{tabular}

165

166

${ }^{*)}$ Extent of Reaction

167

168 

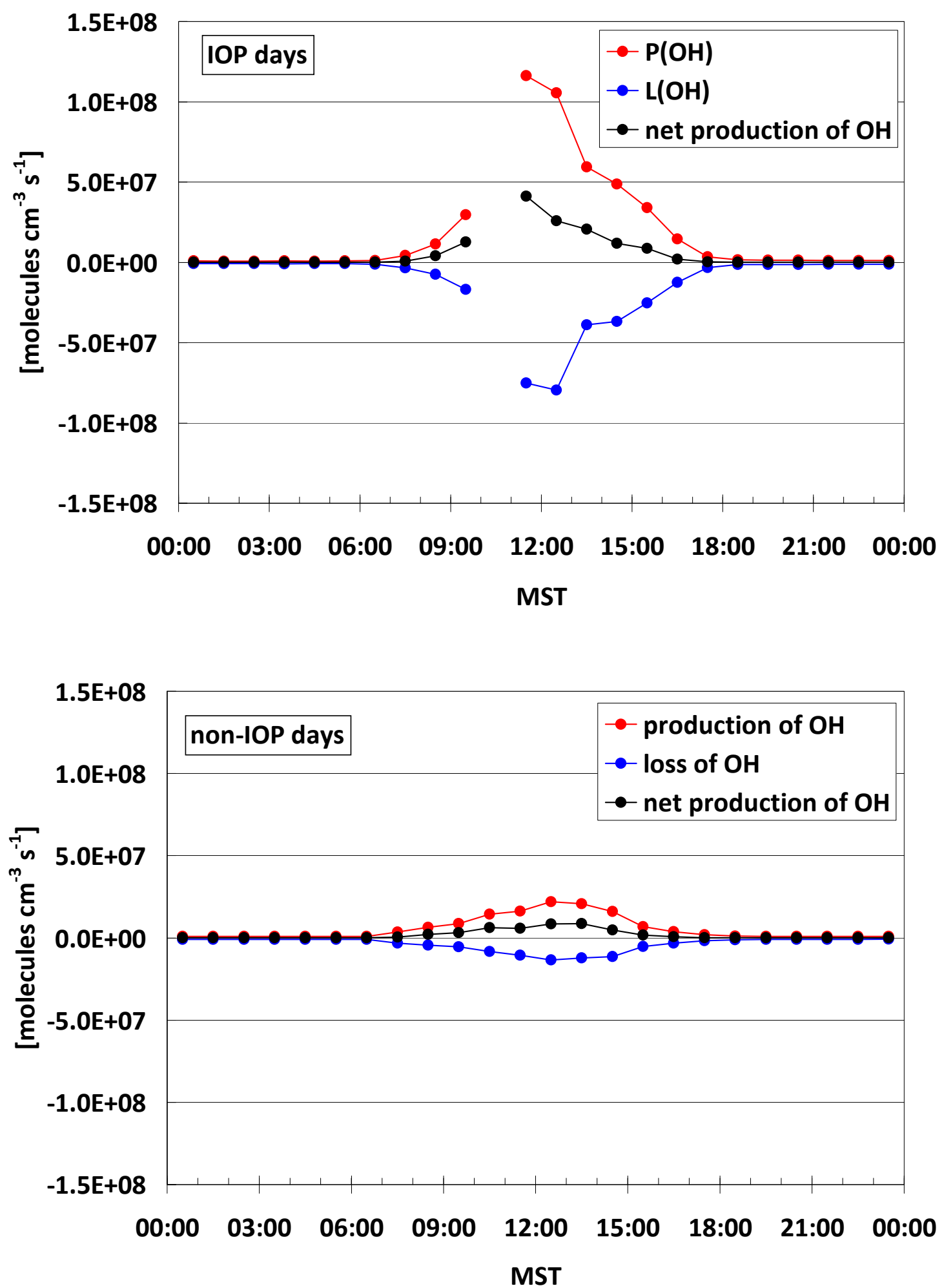

171 S11. Calculated production and loss rates of $\mathrm{OH}$ production, as well as net production rate of 172 OH on IOP and non-IOP days. 
181
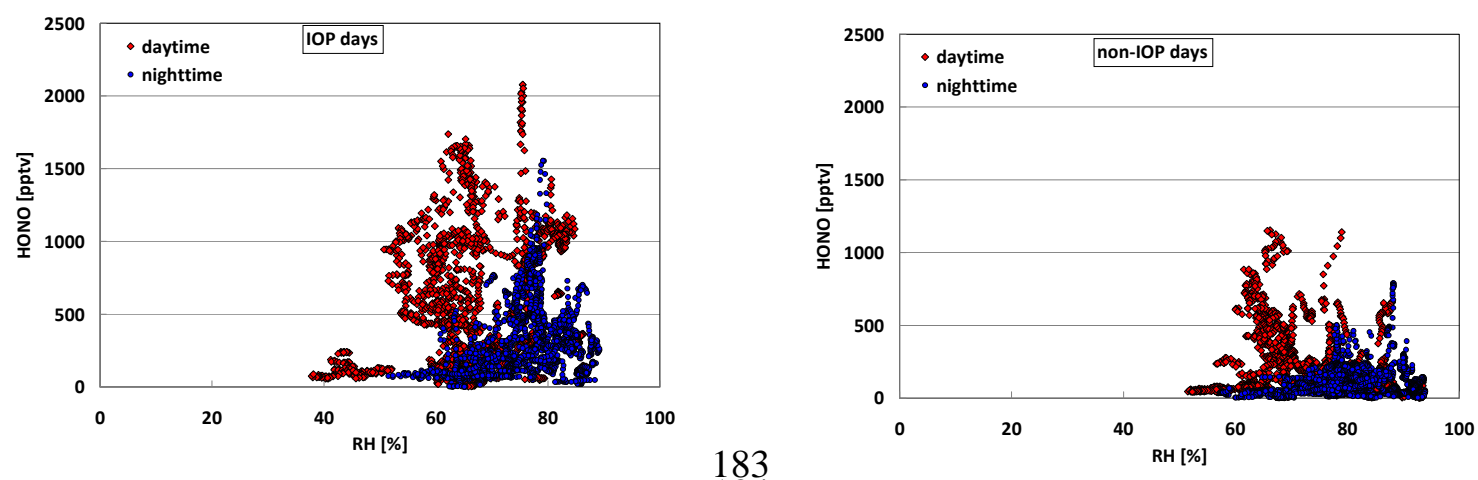

186 S12. HONO mixing ratios versus relative humidity for day- and night-time conditions on IOP

187 and non-IOP days (night-time defined as time periods with solar radiation less than $1 \mathrm{~W} / \mathrm{m}^{2}$ ).

188

189

190

191

192 


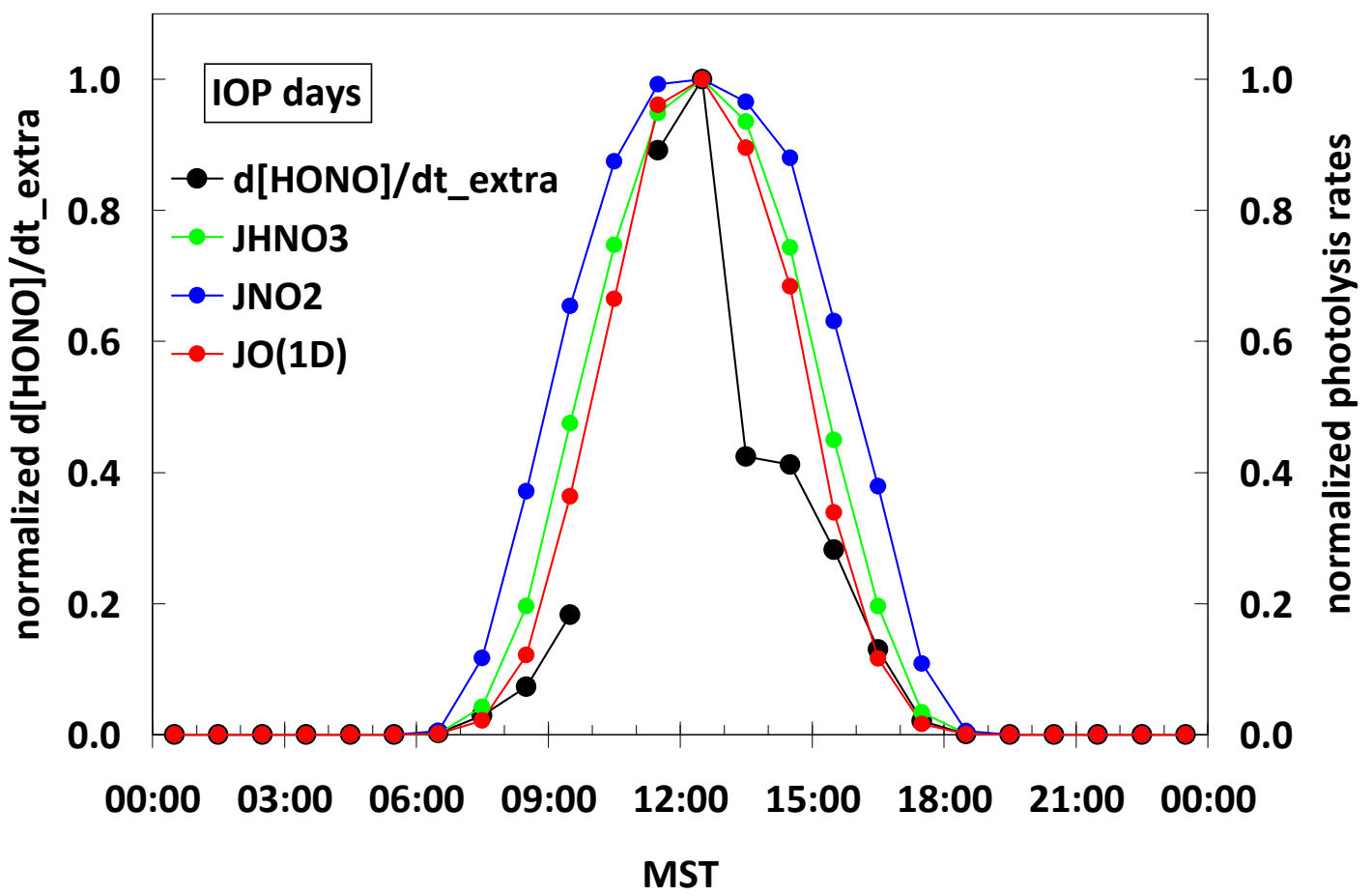

193

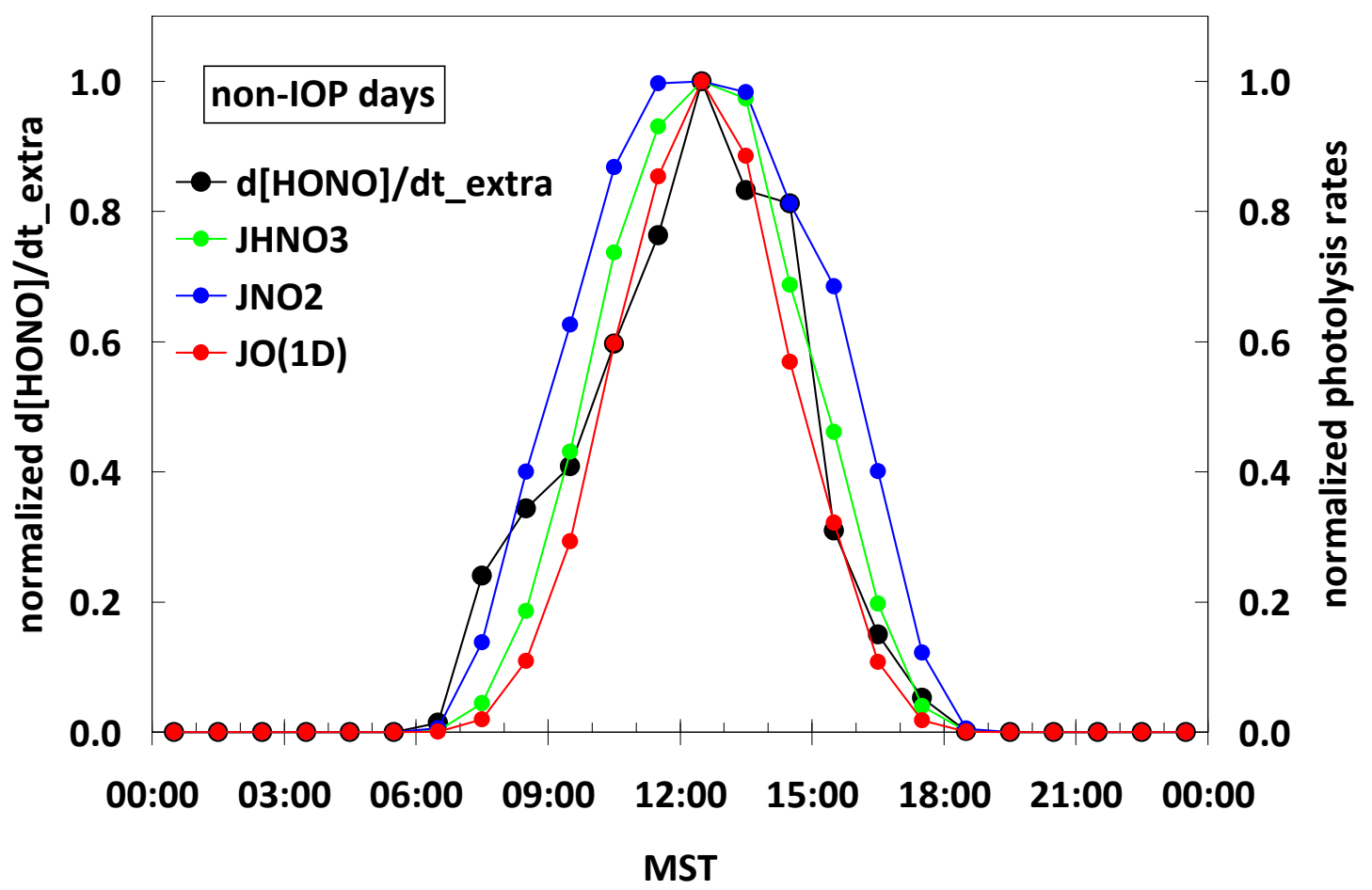

194

195 S13. Median diurnal variation of HONO extra source and $J_{\mathrm{HNO}}, J_{\mathrm{NO} 2}$, and $J_{\mathrm{O}(1 \mathrm{D})}$, normalized 196 to their corresponding daily maximum median value on IOP and non-IOP days. 


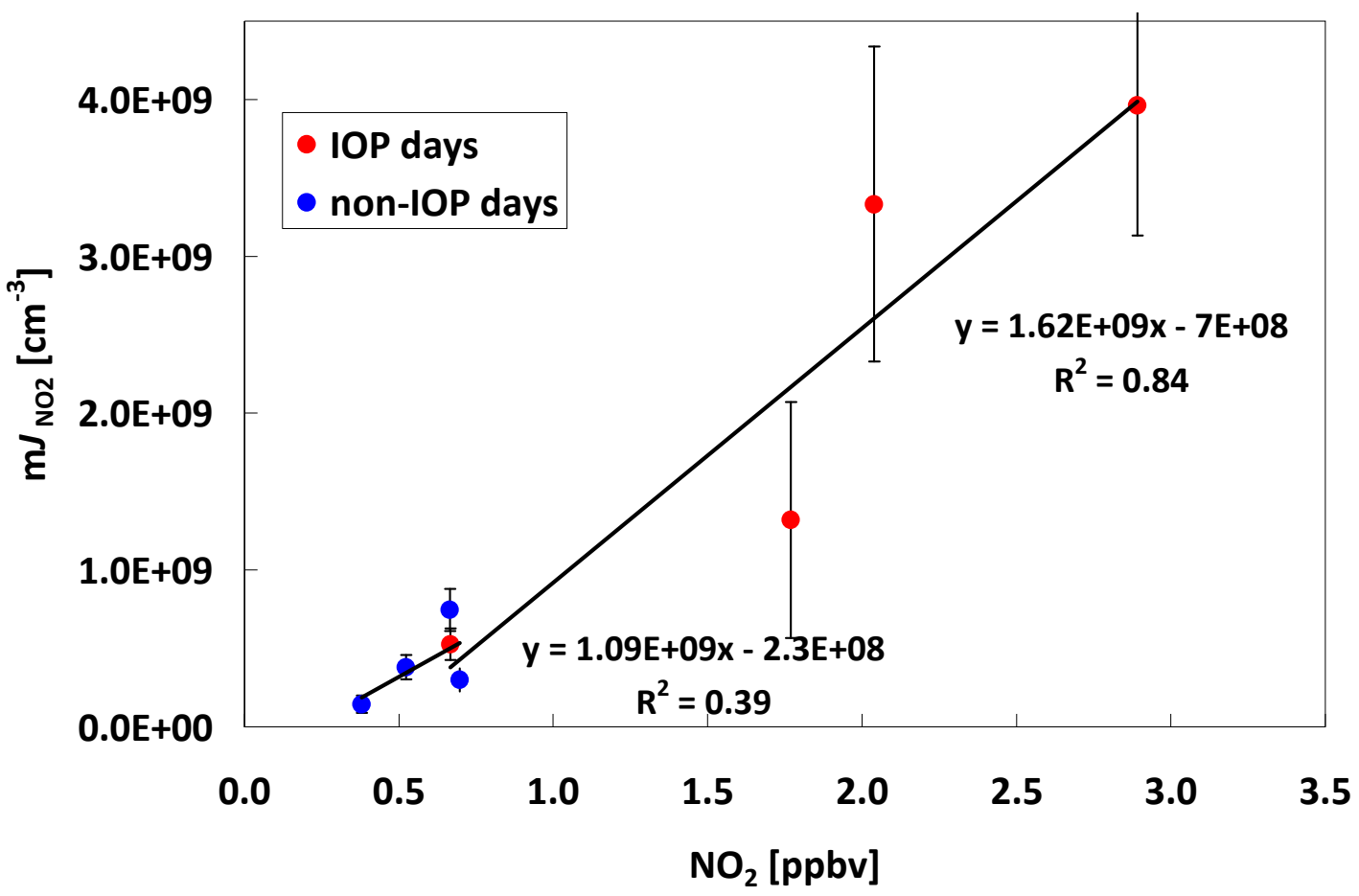

197

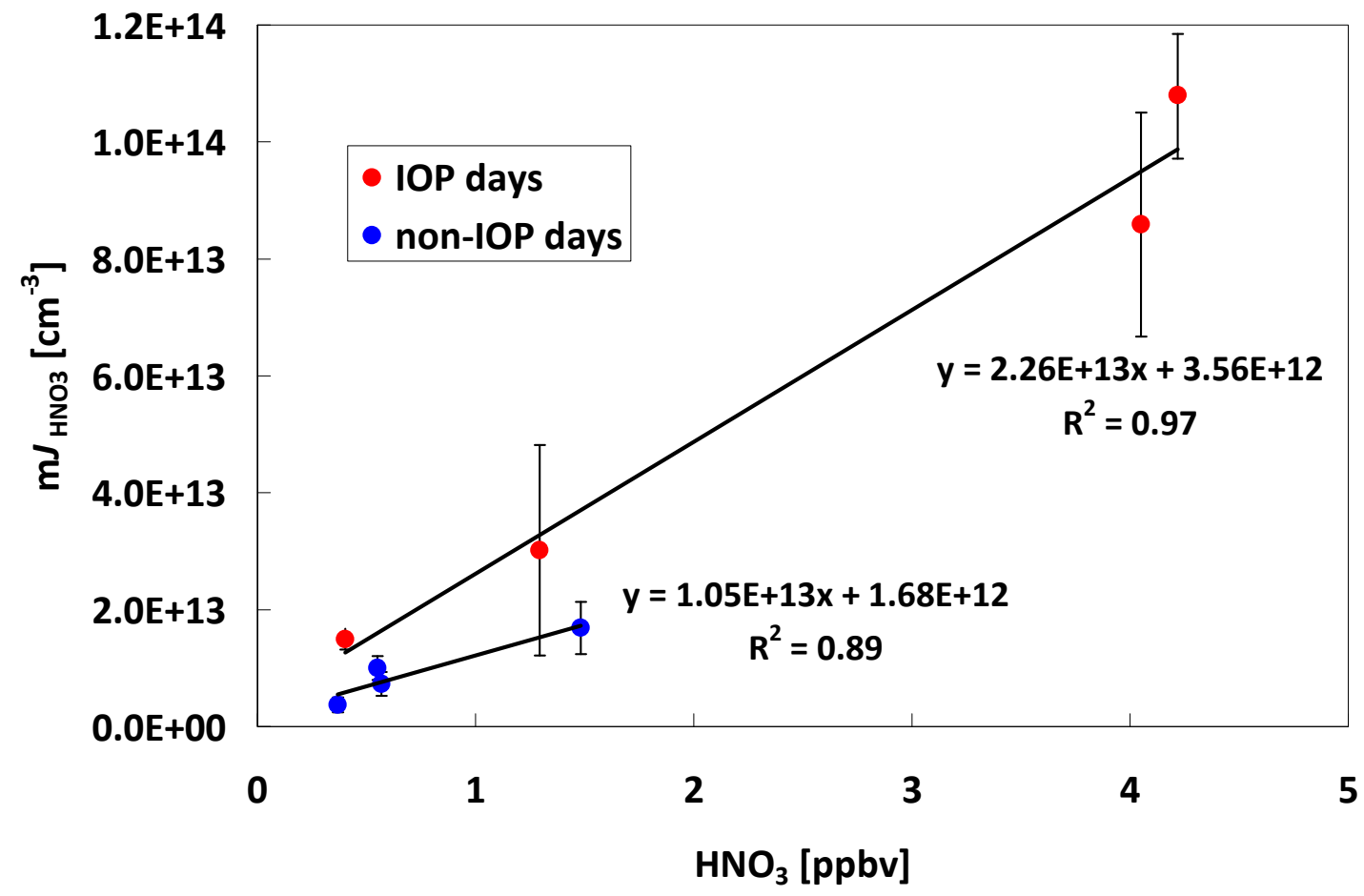

198

199 S14. Correlations of $\mathrm{m} J_{\mathrm{NO} 2}$ and $\mathrm{m} J_{\mathrm{HNO} 3}$ versus daily median $\mathrm{NO}_{2}$ and $\mathrm{HNO}_{3}$ mixing ratios on 200 IOP and non-IOP days. Days with daily median NO mixing ratios than 500 pptv were 201 excluded. 